\title{
Sole Mate: Safe Path-Finding by Obstacle Detection and Distance Estimation for the Blind
}

\author{
Abhigna. R, Amith K Shinde, Ashwin Sundaresh, Dheeraj. P. R, P.A.Vijaya
}

\begin{abstract}
The world has increased its demand for assistive technology (AT). There are a lot of researches and developments going on with respect to AT. Among the AT devices which are being developed, the need for a reliable and less expensive device which serves as an assistance for a visually challenged person is in serious demand all around the world. We, therefore, intend to provide a solution for this by constructing a device that has the capability to detect the obstacles within a given range for a visually challenged person and alerting the person about the obstacles. This involves various components like a camera for image detection, an ultrasonic distance sensor for distance estimation and a vibration motor which works on the principle of Haptic feedback and rotates with varied intensities depending on how far the obstacle is from the user. This paper presents a model which is a part of the footwear of the user and hence, no additional device is required to hold onto for assistance. The model involves the use of a microcontroller, a camera, to dynamically perceive the obstacles and a haptic feedback system to alert the person about the same. The camera dynamically acquires the real time video footage which is further processed by the microcontroller to detect the obstacles. Simultaneously, one more algorithm is being executed to estimate the distance with the help of an ultrasonic distance sensor. Depending on the distance, the frequency of the vibration motor, which acts as the output for notifying the user about the obstacle, is varied (haptic feedback). With this system, a visually challenged person will be able to avoid the obstacles successfully without the use of any additional device.
\end{abstract}

Index Terms - Assistive technology, distance estimation, object detection, visually impaired.

\section{INTRODUCTION}

At present, an efficient reintegration of differently-abled people in the society is highly required. This is possible only if their frailties are assisted or completely replaced. Assistive technology is strongly needful to help in neutralizing these impairments.

Current researches and developments are implementing the recent advancements in embedded systems and image processing techniques in their real time applications to come

Revised Manuscript Received on April 12, 2019.

Abhigna. R, Department of Electronics and Communication Engineering, BNM Institute of Technology, Bengaluru (abhigna.ramamohan@gmail.com)

Amith K Shinde, Department of Electronics and Communication Engineering, BNM Institute of Technology, Bengaluru (amithk.shinde@gmail.com)

AshwinSundaresh, Department of Electronics and Communication Engineering, BNM Institute of Technology, Bengaluru (ashwinsundaresh@gmail.com)

Dheeraj. P. R, Department of Electronics and Communication Engineering, BNM Institute of Technology, Bengaluru (dheerajpr97@gmail.com)

Dr.P.A.Vijaya, Professor and Head, Department of Electronics and Communication Engineering, BNM Institute of Technology, Bengaluru (pavmkv@gmail.com) up with a cost efficient portable assistive device to help the visually challenged. With the present technology, there are many assistive devices in the market that are able to detect obstacles, but, unfortunately, intrusiveness, size and the complexity of the devices prevents the users from taking advantage of them [1].

This paper aims at the design and implementation of a detachable unit which is robust and user friendly, in addition to being cost efficient, thus, trying to aggrandize the functionality of the existing white cane, to concede obstacle detection. The paper elucidates the method of using a camera for detecting the obstructions faced in real time. It uses a haptic feedback system to alert the user about the position of the obstacle.

\section{MOTIVATION}

According to the statistics given by the World Health Organization (WHO), there are around 285 million people who are visually challenged worldwide. Table 1 shows a summary of the detailed report by WHO.

Visually challenged people face many glitches in their daily life, among which, the challenge of independent mobility and aidless navigation is eminent.

\begin{tabular}{|c|c|c|c|c|}
\hline $\begin{array}{c}\text { Ages } \\
\text { (in years) }\end{array}$ & $\begin{array}{c}\text { Population } \\
\text { (millions) }\end{array}$ & $\begin{array}{c}\text { Blind } \\
\text { (millions) }\end{array}$ & $\begin{array}{c}\text { Low Vision } \\
\text { (millions) }\end{array}$ & $\begin{array}{c}\text { Visually } \\
\text { Impaired } \\
\text { (millions) }\end{array}$ \\
\hline $0-14$ & $1,848.50$ & 1.421 & 17.518 & 18.939 \\
\hline $15-49$ & $3,548.20$ & 5.784 & 74.463 & 80.248 \\
\hline$\geq 50$ & $1,340.80$ & 32.160 & 154.043 & 186.203 \\
\hline All ages & $6,737.50$ & 39.365 & 246.024 & 285.389 \\
\hline
\end{tabular}

Table 1. WHO report on world's visually challenged population

A combination of two techniques is used for the mobility of blind and low vision individuals: local navigation and way- finding.

The local navigation technique is basically about relying on external sources like white canes or guide dogs, sounds heard in the surroundings, sensing tactile clues from the neighboring surfaces.

On the other hand, Way-finding technique is quite an unorthodox method which is developed by a matter of experience usually. 
There are a few individuals who increase their wayfinding ability through training. Visually challenged people usually rely on assistance of sighted people to find their way or need an accompanying person to follow during the training period. This means that the majority of the visually challenged people cannot find their without any aid in an unfamiliar area.

At present, the most widely used AT device is a white cane which is a purely mechanical device dedicated to detect static obstacles on the pathway like holes in roads, uneven surfaces, steps and other hazards by the use of a simple tactile-force feedback. Granted these canes are capable of detecting obstacles, it has a low rate feedback system. Thus, the visually challenged find it difficult to navigate in unknown surroundings even with the usage of the available devices. Hence, we have come up with an innovative solution which shall greatly benefit the visually challenged users, and also in a way provide a lifeline for them [2], [3].

\section{METHODOLOGY}

In this Section, we briefly explain the working of the prototype designed.

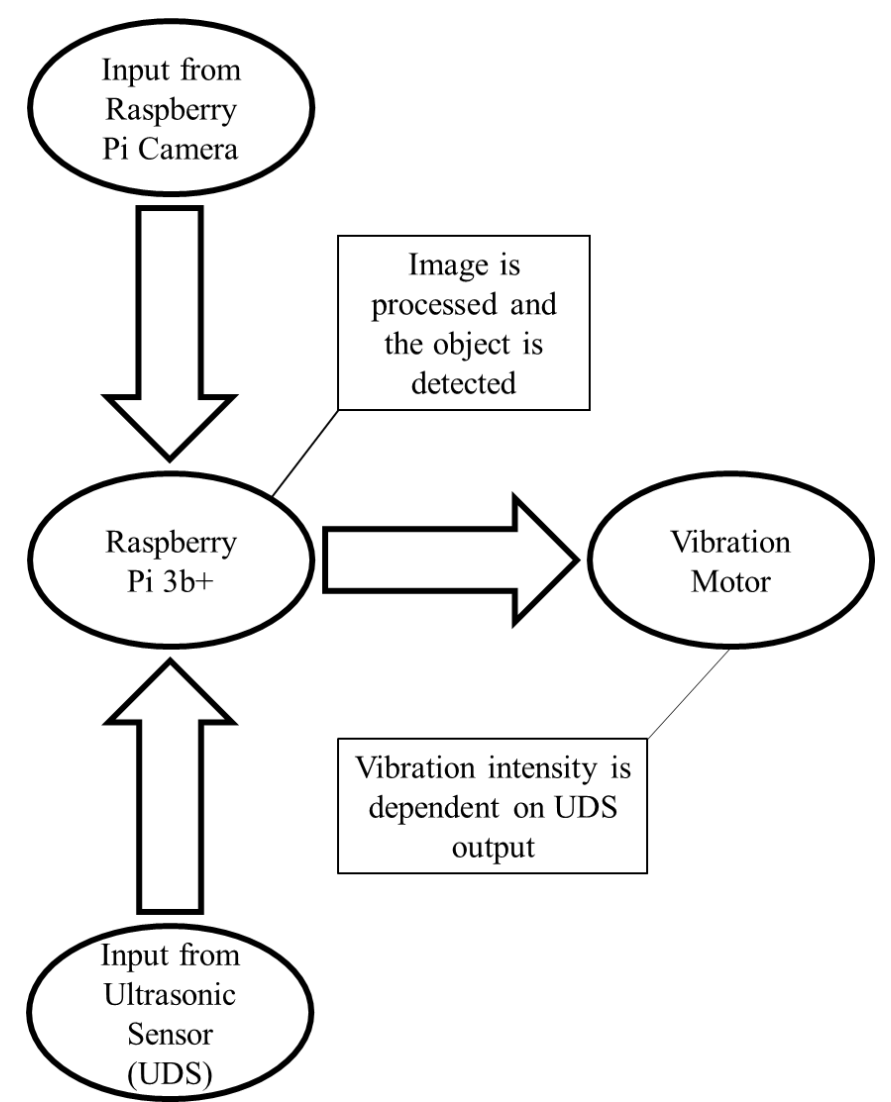

Fig. 1. Working of the prototype

This paper gives an overview on obstacle detection using image processing techniques. From Figure 1, we can observe that the Raspberry Pi camera and Ultrasonic Distance Sensor (UDS) are the inputs to the Raspberry Pi module and the vibration motor is the output device.

Basically, Raspberry $\mathrm{Pi} 3 \mathrm{~B}+$ is interfaced with a Raspberry Pi camera externally. The image captured by the Pi camera is processed and obstacles are detected if present [4]. The algorithm for obstacle detection uses a TensorFlow model which we have discussed in IV Section of this paper.
In case obstacle(s) is detected, the vibration motor gets activated, warning the user about the obstacle(s) near him/her.

In addition to this, we have used an UDS is used to estimate distance. Haptic feedback is implemented such that the feedback, which is in the form of vibrations, varies based on whether the UDS detects the obstacle or not. The intensity of vibrations increases if the UDS detects the obstacle.

Thus, the proposed prototype assists a visually challenged person in such a way that the person can detect the distance of the obstacle by feeling the intensity of the vibrations generated.

The vibration we have used in the prototype is a coin vibration motor of $10 \times 3 \mathrm{~mm}$ size.

We have used a regular power bank to power up the entire setup so make the design as less complex as possible. Using a power bank for power supply requirements also reduces the overall weight of the prototype.

\section{OBSTACLE DETECTION}

In this Section, we explore one of the TensorFlow models, SSD model, to implement obstacle detection algorithm. The specialty of using this model is that multiple objects can be detected simultaneously in a single frame. Also, confidence percentage of the object detected is also displayed [5].

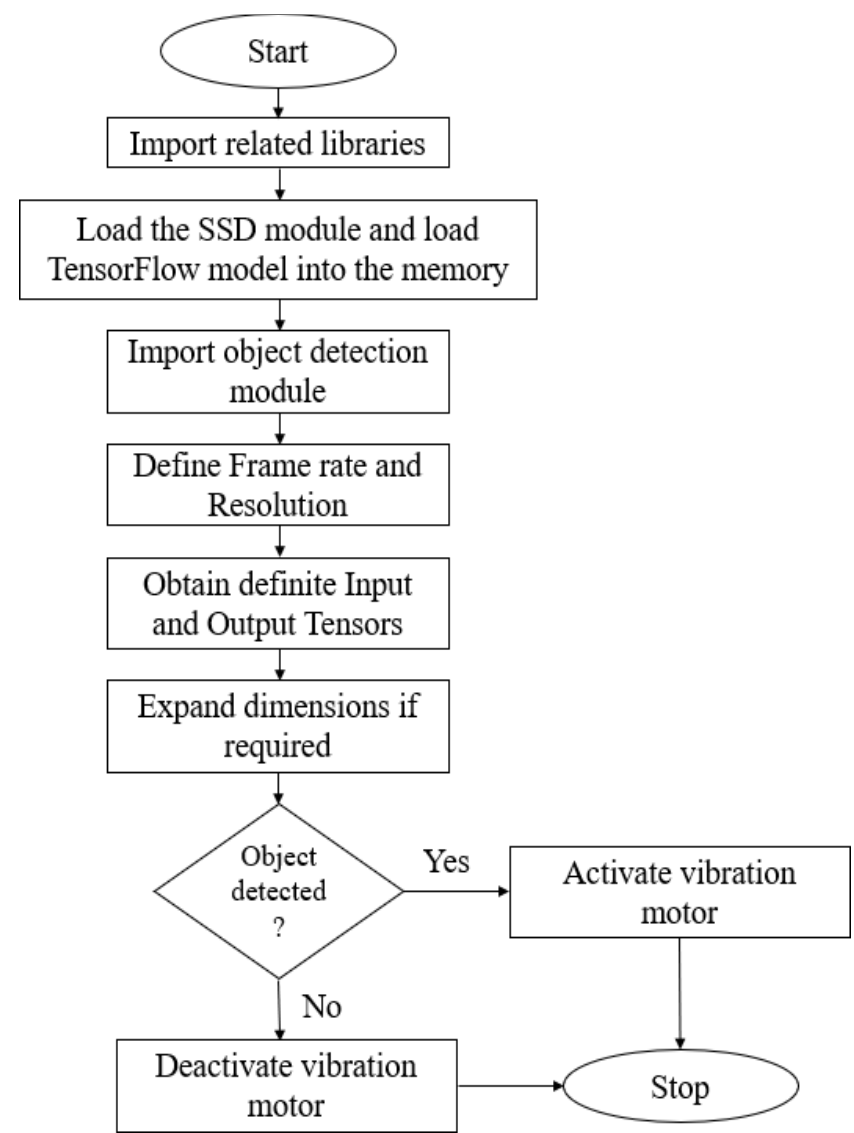

Fig. 2. Flowchart for Obstacle Detection 
The algorithm implemented is as follows:

Step 1: Start

Step 2: Import related libraries and setup virtual environment to allow parallel computing of multiple versions of different modules.

Step 3: Import the object detection module and move on to the model preparation part. By default, "SSD with Mobilenet" model is used in this approach.

Step 4: Download the SSD model and load the TensorFlow model into memory.

Step 5: Import OpenCV library and define Frame rate and Resolution for the captured video.

Step 6: Obtain definite input and output Tensors for the captured video (Each output box/Tensor represents a part of the image where a particular object has been detected).

Step 7: Expand dimensions of the computed image if necessary.

Step 8: Activate vibration motor if obstacle is detected.

Step 9: Exit

Figure 2 represents the flowchart for detecting obstacle using SSD model algorithm. This algorithm also shows the category to which the obstacle belongs to, viz., a person, a car, a truck, a tree or a wall, etc., in the form of confidence percentage. However, a minor uncertainty with respect to the category to which the obstacle belongs to does exist. The vibration motor remains activated until the Raspberry Pi camera can no longer detect any obstacle. If there is no obstacle present, the vibration motor gets deactivated.

\section{DISTANCE ESTIMATION USING UDS}

This Section Distance is estimated using an ultrasonic distance sensor [6] and a vibration motor as input and output respectively.

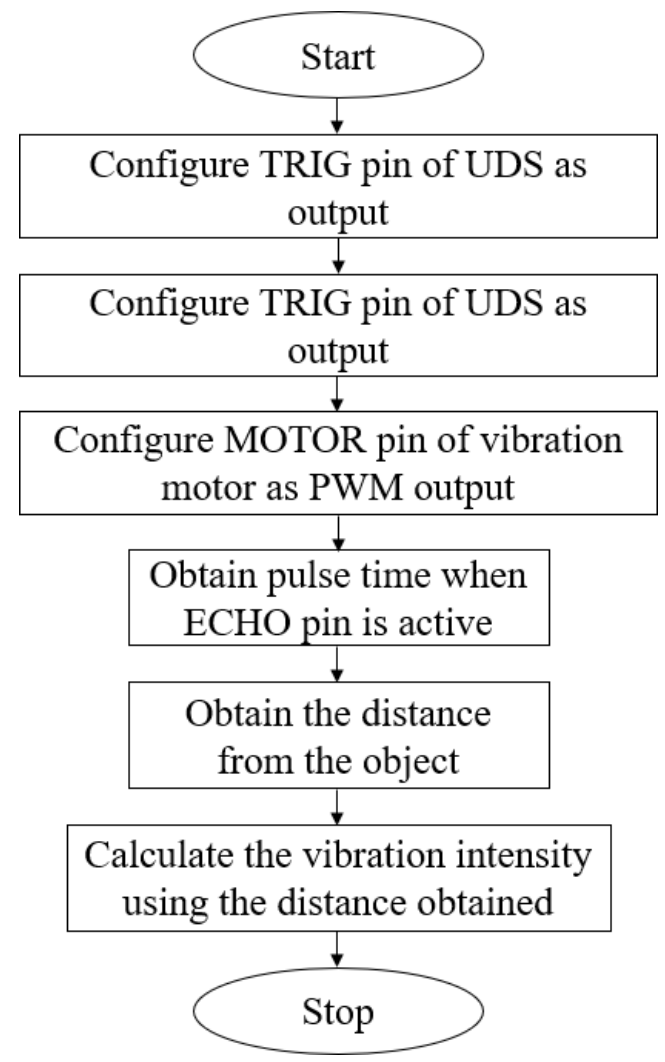

Fig. 3. Flowchart for Distance Estimation
The algorithm implemented to control the UDS and the vibration motor is as follows:

Step 1: Start

Step 2: Configure TRIG and ECHO pins of UDS to function as output and input devices, respectively. Also, configure MOTOR pin of the vibration motor to drive it as a PWM output device.

Step 3: Obtain the pulse time when the ECHO pin is active. Step 4: Calculate the distance of the sensor from an object. Step 5: Vary the vibration intensity using the distance obtained.

\section{Step 6: Exit}

Distance is estimated and the intensity of the vibration motor is varied with the help of this algorithm. Figure 3 shows the flowchart of the algorithm which has been implemented.

A potential divider is used to step down the voltage from the UDS pins to the GPIO pins of the Raspberry Pi module. The values used in the potential divider varies with the Raspberry Pi module being used and the type of power supply being used. Figure 4 shows the potential divider used for our prototype.

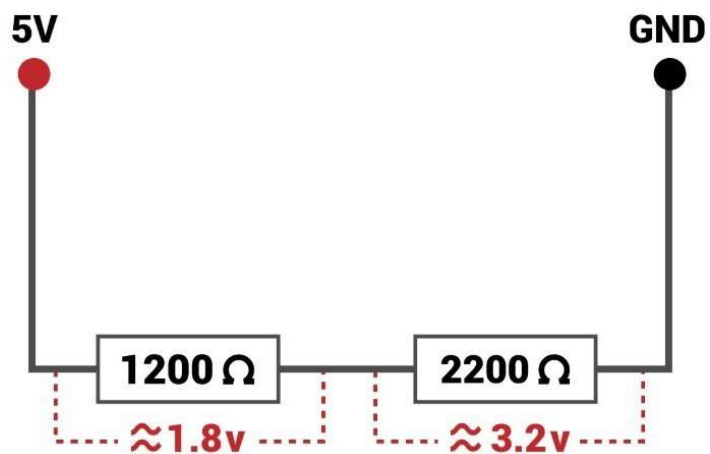

Fig. 4. Potential Divider

\section{EXPERIMENTAL RESULTS}

We developed the codes on python which were based on the algorithms discussed in Section IV and Section V, which were executed on Raspbian OS. The execution occurred in real time where the prototype was kept at the feet of a person, i.e., at the ground level. The prototype was used on a regular road and not in a controlled environment for effective analyzation.

The results obtained when the algorithms were implemented in real time are as follows:

\section{Obstacle detection using SSD model}

We can observe two main points from Figure 5:

(i) Multiple objects (more than three objects) are detected in a single frame.

(ii) The confidence level of the detected obstacles are also displayed in the same frame. 


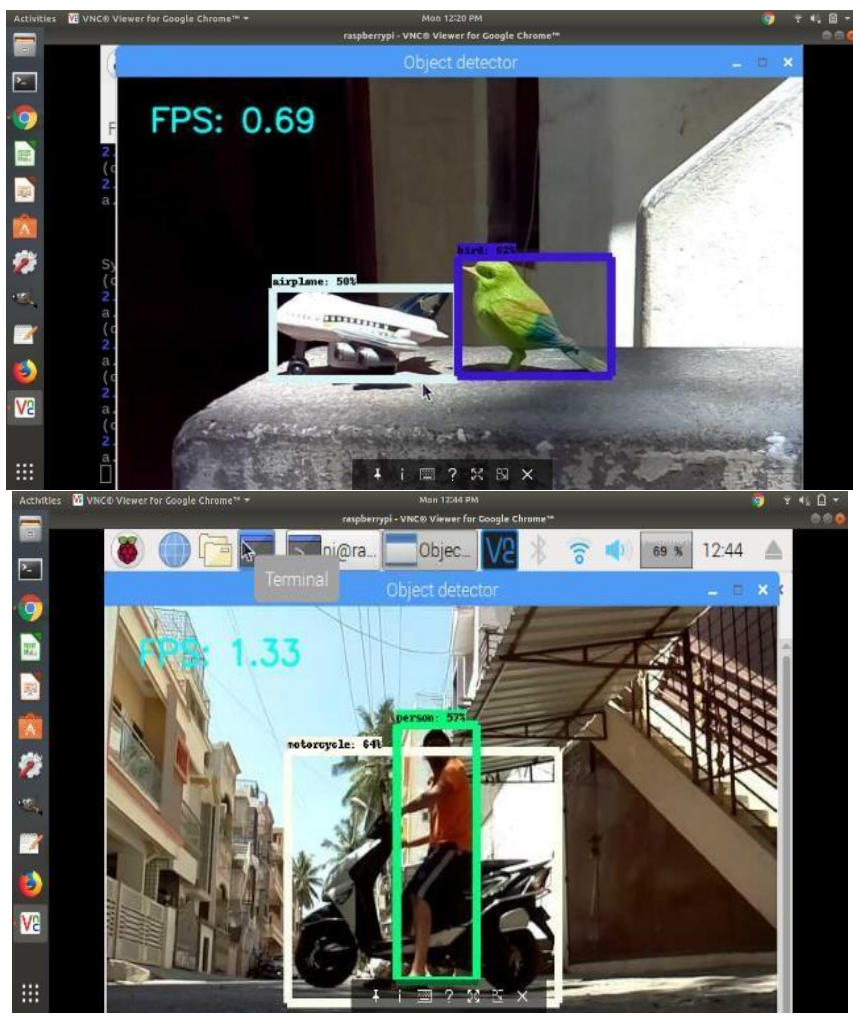

Fig. 5. Output for the Obstacle Detection Algorithm (Short Range)

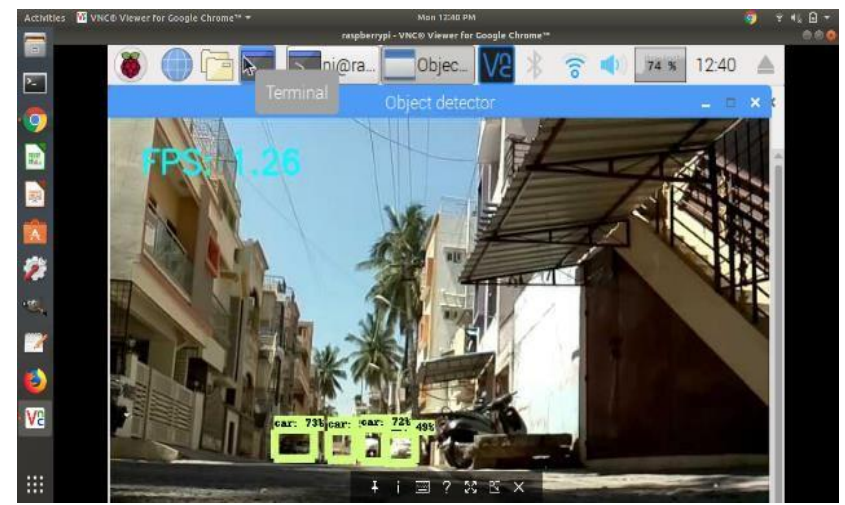

Fig. 6. Output for the Obstacle Detection Algorithm (Long Range)

We can observe an additional feature from Figure 6:

(iii) Obstacles as far as $7 \mathrm{~m}$ to $8 \mathrm{~m}$ are being detected with more than $50 \%$ confidence.

The execution of the algorithm in real time took around 3 seconds, which is quite efficient for a range of $7 \mathrm{~m}$ to $8 \mathrm{~m}$. We can conclude from the above results that the implementation of SSD model for obstacle detection has been successful and can be used for real time applications.

\section{Distance Estimation using UDS}

The UDS we used has a range of $2 \mathrm{~m}$. In real time, however, we observed that the sensor could detect obstacles as far as $180 \mathrm{~m}$ approximately, which is illustrated in Figure 7. In the figure, we can observe that the longest distance detected bythe sensor is $176.8 \mathrm{~m}$.

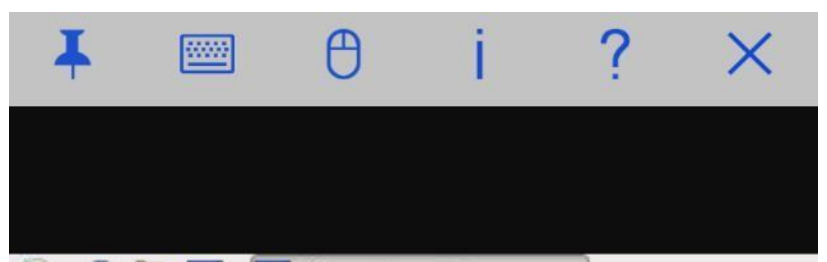

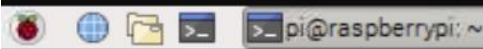

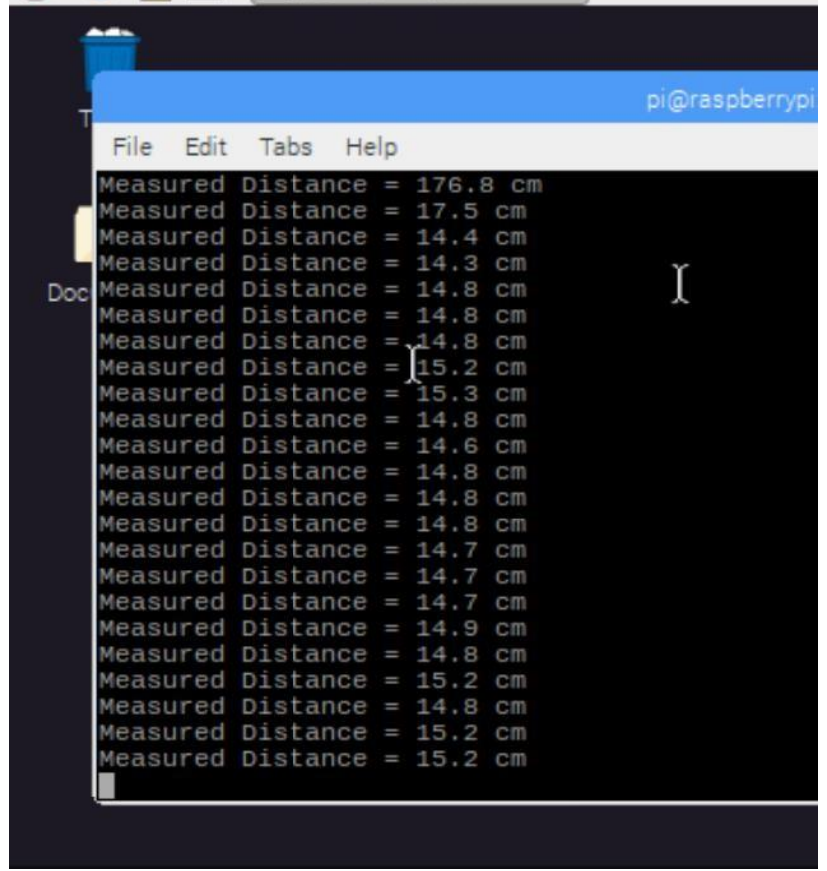

Fig. 7. Output for the Distance Estimation Algorithm

The time taken for the execution of this algorithm in real time is lesser than the obstacle detection algorithm and hence, the user will be warned about the obstacle almost instantaneously if the obstacle is too close.

Experimental results summary:

(i) The results obtained in real time prove that the output is generated almost instantaneously.

(ii) The distance estimated had an accuracy of more than $90 \%$.

(iii) The confidence level of all the objects detected had relatively high level of accuracy at a shorter range and had more than $50 \%$ accuracy in a long range.

\section{CONCLUSION}

The possibilities of exploiting the available technology to solve real world problems are immense. This paper proposes a cost efficient, user-friendly, portable system for obstacle detection and distance estimation.

In this paper, we have discussed the applications of object detection and distance estimation concepts in apprising a visually challenged person about the obstacles around him. An overview on the working of the prototype is elucidated, based on which algorithms are written. The algorithm-based codes for obstacle detection and distance estimation are executed and the results are discussed.

We observed that the two algorithms can be run simultaneously in real time where the outputs are generated without much delay.

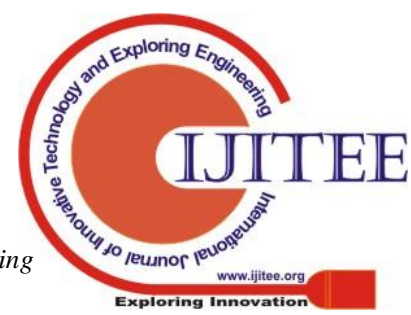




\section{FUTURE SCOPE}

The present prototype is limited to linear range of obstacle detection. An angular positioning of the Raspberry Pi camera or using multiple Raspberry $\mathrm{Pi}$ cameras can expand the range of obstacle detection. Also, to make it more time efficient, the SSD model can be replaced with a model based on neural networks for safe path detection may be implemented. This increases the object detection ability of the prototype and will decrease the need for manual inputs, making the system more machine oriented. In addition to safe path detection, to assure that the user can contact someone in case of emergency, a GSM module can be implemented to locate the user in case of an emergency.

\section{REFERENCES}

1. ZeeshanSaquib, VishakhaMurari, Suhas N Bhargav, "BlinDar: An invisible eye for the blind people making life easy for the blind with Internet of Things (IoT)", 2nd International Conference on Recent Trends in Electronics, Information and Communication Technology (RTEICT), May, 2017.

2. NiranjanDebnath, ZulAziziHailani, SakinahJamaludin, Syed Abdul Kader Aljunid, "An Electronically Walking Guided Stick for Blind", Proceedings of the 23rd Annual EMBS International Conference, 2001.

3. Mohammad FaridSaaid, Ismarani Ismail, MohmdZikrul Hakim Noor, "Radio Frequency Identification Walking Stick (RFIWS)- A Device for the Blind", \%th International Colloquium on Signal Processing and its Application (CSPA), 2009.

4. Vijayalaxmi, K Anjali, B Srujana, P Rohith Kumar, "Object Detection and Tracking using Image Processing", Global Journal of Advanced Engineering Technologies, Special Issue (CTCNSF-2014).

5. Redmon J, Divvala S, Girshick R, Farhadi A, "You Only Look Once: Unified, Real-Time Object Detection, In:CVPR, 2016.

6. JaroslawMajchrzak, Mateusz Michalski, GrzergorzWiczynski, "Distance Estimation with a Long-Range Ultrasonic Sensor System”, IEEE Sensors Journal, Vol.9, Issue No.7, July, 2009. 